\title{
Pareto Near-Optimal Strategy of Multimodeling Systems
}

\author{
Hiroaki Mukaidani \\ Hiroshima City University \\ 3-4-1, Ozuka-Higashi, Asaminami-ku, Hiroshima, 731-3194 Japan \\ E-mail mukaida@im.hiroshima-cu.ac.jp \\ URL http://www.cntl.im.hiroshima-cu.ac.jp/ mukaida/
}

\begin{abstract}
In this paper, we study the Pareto near-optimal strategy for multiparameter singularly perturbed system (MSPS). The main contribution is to propose a new method for obtaining the Pareto near-optimal strategy. We show that the resulting near-optimal strategy achieves the cost functional $J_{j}^{*}+O(\|\mu\|)$. Moreover, we also show that the resulting Pareto near-optimal strategy is equivalent to the existing composite strategy.
\end{abstract}

\section{INTRODUCTION}

Multimodeling stability, control and filtering problems have been investigated extensively (see e.g., [1]-[5]). The multimodeling problems arise in large scale dynamic systems. For example, these multimodel situations in practice are illustrated by the multi-area power system [1]. A popular approach to deal with the multiparameter singularly perturbed systems (MSPS) is the two-time-scale design method [1]-[5]. When the positive parameters $\varepsilon_{j}, j=1,2$ are very small or unknown the previously used technique is very efficient. However, in order to obtain the slow subsystem, the nonsingularity of the fast state matrices are needed.

In this paper, we study the Pareto near-optimal strategy for the MSPS. We first investigate the uniqueness and boundedness of the solution to the multiparameter algebraic Riccati equation (MARE) and establish its asymptotic structure. The proof of the existence of the solution to the MARE with asymptotic expansion is obtained by an implicit function theorem. This paper presents an improvement on some of the results of [4] in the sense that there is no assumption for the nonsingularlity of the fast state matrices. We also investigate the stabilizability and detectability for the reduced-order algebraic Riccati equation (ARE). The main contribution is to propose a new method for obtaining the Pareto near-optimal strategy. We show that the proposed strategy achieves the cost functional $J_{j}^{*}+O(\|\mu\|), \mu=\left[\begin{array}{ll}\varepsilon_{1} & \varepsilon_{2}\end{array}\right]$ even if $A_{j j}$ is nonsingular, where $J_{j}^{*}$ is the optimal cost. Thus, our new results are applicable to more realistic MSPS compared with [1]. Moreover, when $A_{j j}$ is nonsingular, we also show that the resulting Pareto near-optimal strategy is equivalent to the existing composite strategy [1]. Therefore, we claim that the new Pareto near-optimal strategy includes the existing one [1] as a special case.

\section{Pareto Optimal Strategy}

We consider the linear time-invariant MSPS

$$
\begin{aligned}
\dot{x}_{0}(t) & =A_{00} x_{0}(t)+A_{01} x_{1}(t)+A_{02} x_{2}(t) \\
& +B_{01} u_{1}(t)+B_{02} u_{2}(t), x_{0}(0)=x_{0}^{0}, \\
\varepsilon_{1} \dot{x}_{1}(t) & =A_{10} x_{0}(t)+A_{11} x_{1}(t)+B_{11} u_{1}(t), x_{1}(0)=x_{1}^{0}(1 \mathrm{~b}) \\
\varepsilon_{2} \dot{x}_{2}(t) & =A_{20} x_{0}(t)+A_{22} x_{2}(t)+B_{22} u_{2}(t), x_{2}(0)=x_{2}^{0},(1 \mathrm{c})
\end{aligned}
$$

where $x_{j} \in \mathbf{R}^{n_{j}}, j=0,1,2$ are the state vector, $u_{j} \in$ $\mathbf{R}^{m_{j}}, j=1,2$ are the control input. All the matrices are constant matrices of appropriate dimensions.

$\varepsilon_{1}$ and $\varepsilon_{2}$ are two small positive singular parameters of the same order of magnitude such that

$$
0<k_{1} \leq \alpha \equiv \frac{\varepsilon_{1}}{\varepsilon_{2}} \leq k_{2}<\infty
$$

Note that the fast state matrices $A_{j j}, j=1,2$ may be singular. In the Pareto optimal strategy of the above MSPS (1), a quadratic cost functional is given by

$$
J_{j}=\frac{1}{2} \int_{0}^{\infty}\left[z_{j}^{T}(t) z_{j}(t)+u_{j}^{T}(t) R_{j} u_{j}(t)\right] d t, \quad j=1,2
$$

where $z_{j}(t)=C_{j 0} x_{0}(t)+C_{j j} x_{j}(t) \in \mathbf{R}^{r_{j}}, j=0,1,2$.

A Pareto solution is a pair $u_{1}, u_{2}$ which minimizes

$$
J=\gamma_{1} J_{1}+\gamma_{2} J_{2}, 0<\gamma_{j}<1, \gamma_{1}+\gamma_{2}=1
$$

for some $\gamma_{1}$ and $\gamma_{2}$. It is well known from [1] that the solution of the Pareto optimal strategy is given by

$$
u_{j}^{*}(t)=-\frac{1}{\gamma_{j}} R_{j}^{-1} B_{j \mathcal{E}}^{T} P_{\mathcal{E}} x(t), j=1,2,
$$

where $P_{\mathcal{E}}$ satisfies the MARE

$$
A_{\mathcal{E}}^{T} P_{\mathcal{E}}+P_{\mathcal{E}} A_{\mathcal{E}}-P_{\mathcal{E}} S_{\mathcal{E}} P_{\mathcal{E}}+Q=0
$$

with

$$
\begin{aligned}
& A_{\mathcal{E}}=\left[\begin{array}{ccc}
A_{00} & A_{01} & A_{02} \\
\varepsilon_{1}^{-1} A_{10} & \varepsilon_{1}^{-1} A_{11} & 0 \\
\varepsilon_{2}^{-1} A_{20} & 0 & \varepsilon_{2}^{-1} A_{22}
\end{array}\right], \\
& B_{1 \mathcal{E}}=\left[\begin{array}{c}
B_{01} \\
\varepsilon_{1}^{-1} B_{11} \\
0
\end{array}\right], \quad B_{2 \mathcal{E}}=\left[\begin{array}{c}
B_{02} \\
0 \\
\varepsilon_{2}^{-1} B_{22}
\end{array}\right],
\end{aligned}
$$


IECON'01: The 27th Annual Conference of the IEEE Industrial Electronics Society

$$
\begin{aligned}
Q_{1} & =\left[\begin{array}{ccc}
C_{10}^{T} C_{10} & C_{10}^{T} C_{11} & 0 \\
C_{11}^{T} C_{10} & C_{11}^{T} C_{11} & 0 \\
0 & 0 & 0
\end{array}\right], \\
Q_{2} & =\left[\begin{array}{ccc}
C_{20}^{T} C_{20} & 0 & C_{20}^{T} C_{22} \\
0 & 0 & 0 \\
C_{22}^{T} C_{20} & 0 & C_{22}^{T} C_{22}
\end{array}\right], \\
S_{j \mathcal{E}}= & B_{j \mathcal{E}} R_{j}^{-1} B_{j \mathcal{E}}^{T}, j=1,2, \\
S_{\mathcal{E}}= & \frac{1}{\gamma_{1}} S_{1 \mathcal{E}}+\frac{1}{\gamma_{2}} S_{2 \mathcal{E}}, \quad Q=\gamma_{1} Q_{1}+\gamma_{2} Q_{2}, \\
S_{\mathcal{E}}= & {\left[\begin{array}{ccc}
S_{00} & \varepsilon_{1}^{-1} S_{01} & \varepsilon_{2}^{-1} S_{02} \\
\varepsilon_{1}^{-1} S_{01}^{T} & \varepsilon_{1}^{-2} S_{11} & 0 \\
\varepsilon_{2}^{-1} S_{02}^{T} & 0 & \varepsilon_{2}^{-2} S_{22}
\end{array}\right], } \\
Q= & {\left[\begin{array}{ccc}
Q_{00} & Q_{01} & Q_{02} \\
Q_{01}^{T} & Q_{11} & 0 \\
Q_{02}^{T} & 0 & Q_{22}
\end{array}\right] . }
\end{aligned}
$$

A solution $P_{\mathcal{E}}$ of the MARE (6), if it exists, must contain the parameters $\varepsilon_{j}$ because the matrices $A_{\mathcal{E}}$ and $S_{\mathcal{E}}$ contain the $\varepsilon_{j}^{-1}$-order parameter. Taking into account this fact, we look for a solutions $P_{\mathcal{E}}$ of the MARE (6) with the structure

$$
P_{\mathcal{E}}=\left[\begin{array}{ccc}
P_{00} & \varepsilon_{1} P_{10}^{T} & \varepsilon_{2} P_{20}^{T} \\
\varepsilon_{1} P_{10} & \varepsilon_{1} P_{11} & \sqrt{\varepsilon_{1} \varepsilon_{2}} P_{21}^{T} \\
\varepsilon_{2} P_{20} & \sqrt{\varepsilon_{1} \varepsilon_{2}} P_{21} & \varepsilon_{2} P_{22}
\end{array}\right] \in \mathbf{R}^{N \times N},
$$

where $N=n_{0}+n_{1}+n_{2}, \quad P_{00}=P_{00}^{T}, P_{11}=P_{11}^{T}, P_{22}=P_{22}^{T}$.

A near-optimal Pareto strategy for the MSPS has been proposed in [1]. The algorithm consists of solving three separate subproblems, one in a slow time scale and two in fast time scale, and then combining the solutions of these problems to the specific form of the control law. However, in order to separate the MSPS, the nonsingularity of the matrices $A_{j j}, j=1,2$ are required. To avoid these assumptions we propose a method which is different from the existing method [1].

\section{MARE}

Before we present the Pareto near-optimal strategy, we first study the asymptotic structure for the MARE (6). The MARE (6) can be partitioned into

$$
\begin{aligned}
f_{1}= & A_{00}^{T} P_{00}+P_{00} A_{00}+A_{10}^{T} P_{10}+P_{10}^{T} A_{10} \\
& +A_{20}^{T} P_{20}+P_{20}^{T} A_{20}-P_{00} S_{00} P_{00}-P_{10}^{T} S_{01}^{T} P_{00} \\
& -P_{00} S_{01} P_{10}-P_{20}^{T} S_{02}^{T} P_{00}-P_{00} S_{02} P_{20} \\
& -P_{10}^{T} S_{11} P_{10}-P_{20}^{T} S_{22} P_{20}+Q_{00}=0, \\
f_{2}= & P_{00} A_{01}+P_{10}^{T} A_{11}+\varepsilon_{1} A_{00}^{T} P_{10}^{T}+A_{10}^{T} P_{11}+\sqrt{\alpha} A_{20}^{T} P_{21} \\
& -\varepsilon_{1}\left(P_{00} S_{00} P_{10}^{T}+P_{10}^{T} S_{01}^{T} P_{10}^{T}+P_{20}^{T} S_{02}^{T} P_{10}^{T}\right) \\
& -P_{00} S_{01} P_{11}-P_{10}^{T} S_{11} P_{11} \\
& -\sqrt{\alpha}\left(P_{00} S_{02} P_{21}+P_{20}^{T} S_{22} P_{21}\right)+Q_{01}=0, \\
f_{3}= & P_{00} A_{02}+P_{20}^{T} A_{22}+\varepsilon_{2} A_{00}^{T} P_{20}^{T}+A_{20}^{T} P_{22}+\frac{1}{\sqrt{\alpha}} A_{10}^{T} P_{21}^{T} \\
& -\varepsilon_{2}\left(P_{00} S_{00} P_{20}^{T}+P_{10}^{T} S_{01}^{T} P_{20}^{T}+P_{20}^{T} S_{02}^{T} P_{20}^{T}\right) \\
& -P_{00} S_{02} P_{22}-P_{20}^{T} S_{22} P_{22} \\
& -\frac{1}{\sqrt{\alpha}}\left(P_{00} S_{01} P_{21}^{T}+P_{10}^{T} S_{11} P_{21}^{T}\right)+Q_{02}=0,
\end{aligned}
$$

$$
\begin{aligned}
f_{4}= & A_{11}^{T} P_{11}+P_{11} A_{11}+\varepsilon_{1}\left(A_{01}^{T} P_{10}^{T}+P_{10} A_{01}\right) \\
& -\varepsilon_{1}\left(\varepsilon_{1} P_{10} S_{00} P_{10}^{T}+P_{11} S_{01}^{T} P_{10}^{T}+\sqrt{\alpha} P_{21}^{T} S_{02}^{T} P_{10}^{T}\right) \\
& -\varepsilon_{1}\left(P_{10} S_{01} P_{11}+\sqrt{\alpha} P_{10} S_{02} P_{21}\right)-P_{11} S_{11} P_{11} \\
& -\alpha P_{21}^{T} S_{22} P_{21}+Q_{11}=0, \\
f_{5}= & \varepsilon_{1} P_{10} A_{02}+\varepsilon_{2} A_{01}^{T} P_{20}^{T} \\
& -\varepsilon_{2}\left(\varepsilon_{1} P_{10} S_{00} P_{20}^{T}+P_{11} S_{01}^{T} P_{20}^{T}+\sqrt{\alpha} P_{21}^{T} S_{02}^{T} P_{20}^{T}\right) \\
& -\varepsilon_{1}\left(P_{10} S_{02} P_{22}+\frac{1}{\sqrt{\alpha}} P_{10} S_{01} P_{21}^{T}\right) \\
& +\sqrt{\alpha} P_{21}^{T}\left(A_{22}-S_{22} P_{22}\right) \\
& +\frac{1}{\sqrt{\alpha}}\left(A_{11}-S_{11} P_{11}\right)^{T} P_{21}^{T}=0, \\
= & A_{22}^{T} P_{22}+P_{22} A_{22}+\varepsilon_{2}\left(A_{02}^{T} P_{20}^{T}+P_{20} A_{02}\right) \\
& -\varepsilon_{2}\left(\varepsilon_{2} P_{20} S_{00} P_{20}^{T}+P_{22} S_{02}^{T} P_{20}^{T}+\frac{1}{\sqrt{\alpha}} P_{21} S_{01}^{T} P_{20}^{T}\right) \\
& -\varepsilon_{2}\left(P_{20} S_{02} P_{22}+\frac{1}{\sqrt{\alpha}} P_{20} S_{01} P_{21}^{T}\right)-P_{22} S_{22} P_{22} \\
& -\frac{1}{\alpha} P_{21} S_{11} P_{21}^{T}+Q_{22}=0 .
\end{aligned}
$$

It is assumed that the limit of $\alpha$ exists as $\varepsilon_{1}$ and $\varepsilon_{2}$ tend to zero [1]-[5], that is

$$
\bar{\alpha}=\lim _{\substack{\varepsilon_{1} \rightarrow+0 \\ \varepsilon_{2} \rightarrow+0}} \alpha .
$$

Let $\bar{P}_{00}, \bar{P}_{10}, \bar{P}_{20}, \bar{P}_{11}, \bar{P}_{21}$ and $\bar{P}_{22}$ be the limiting solutions of the above equation (7) as $\varepsilon_{j} \rightarrow+0, j=1,2$, then we obtain the following equations

$$
\begin{aligned}
& A_{00}^{T} \bar{P}_{00}+\bar{P}_{00} A_{00}+A_{10}^{T} \bar{P}_{10}+\bar{P}_{10}^{T} A_{10}+A_{20}^{T} \bar{P}_{20}+\bar{P}_{20}^{T} A_{20} \\
& \quad-\bar{P}_{00} S_{00} \bar{P}_{00}-\bar{P}_{10}^{T} S_{01}^{T} \bar{P}_{00}-\bar{P}_{00} S_{01} \bar{P}_{10}-\bar{P}_{20}^{T} S_{02}^{T} \bar{P}_{00} \\
& \quad-\bar{P}_{00} S_{02} \bar{P}_{20}-\bar{P}_{10}^{T} S_{11} \bar{P}_{10}-\bar{P}_{20}^{T} S_{22} \bar{P}_{20}+Q_{00}=0 \\
& \bar{P}_{00} A_{01}+\bar{P}_{10}^{T} A_{11}+A_{10}^{T} \bar{P}_{11}+\sqrt{\bar{\alpha}} A_{20}^{T} \bar{P}_{21}-\bar{P}_{00} S_{01} \bar{P}_{11} \\
& \quad-\bar{P}_{10}^{T} S_{11} \bar{P}_{11}-\sqrt{\bar{\alpha}}\left(\bar{P}_{00} S_{02} \bar{P}_{21}+\bar{P}_{20}^{T} S_{22} \bar{P}_{21}\right) \\
& \quad+Q_{01}=0
\end{aligned}
$$

$\bar{P}_{00} A_{02}+\bar{P}_{20}^{T} A_{22}+A_{20}^{T} \bar{P}_{22}+\frac{1}{\sqrt{\bar{\alpha}}} A_{10}^{T} \bar{P}_{21}^{T}-\bar{P}_{00} S_{02} \bar{P}_{22}$

$$
-\bar{P}_{20}^{T} S_{22} \bar{P}_{22}-\frac{1}{\sqrt{\bar{\alpha}}}\left(\bar{P}_{00} S_{01} \bar{P}_{21}^{T}+\bar{P}_{10}^{T} S_{11} \bar{P}_{21}^{T}\right)
$$

$+Q_{02}=0$,

$A_{11}^{T} \bar{P}_{11}+\bar{P}_{11} A_{11}-\bar{P}_{11} S_{11} \bar{P}_{11}$

$-\bar{\alpha} \bar{P}_{21}^{T} S_{22} \bar{P}_{21}+Q_{11}=0$,

$\sqrt{\bar{\alpha}} \bar{P}_{21}^{T}\left(A_{22}-S_{22} \bar{P}_{22}\right)+\frac{1}{\sqrt{\bar{\alpha}}}\left(A_{11}-S_{11} \bar{P}_{11}\right)^{T} \bar{P}_{21}^{T}=0$,

$A_{22}^{T} \bar{P}_{22}+\bar{P}_{22} A_{22}-\bar{P}_{22} S_{22} \bar{P}_{22}$

$$
-\frac{1}{\bar{\alpha}} \bar{P}_{21} S_{11} \bar{P}_{21}^{T}+Q_{22}=0 \text {. }
$$

Assumption 1: The triples $\left(A_{j j}, B_{j j}, C_{j j}\right), j=1,2$ are stabilizable and detectable.

Assumption 2:

$\operatorname{rank}\left[\begin{array}{ccccc}s I_{n_{0}}-A_{00} & -A_{01} & -A_{02} & B_{01} & B_{02} \\ -A_{10} & -A_{11} & 0 & B_{11} & 0 \\ -A_{20} & 0 & -A_{22} & 0 & B_{22}\end{array}\right]=N$, 
IECON'01: The 27th Annual Conference of the IEEE Industrial Electronics Society

$\operatorname{rank}\left[\begin{array}{ccccc}s I_{n_{0}}-A_{00}^{T} & -A_{10}^{T} & -A_{20}^{T} & C_{10}^{T} & C_{20}^{T} \\ -A_{01}^{T} & -A_{11}^{T} & 0 & C_{11}^{T} & 0 \\ -A_{02}^{T} & 0 & -A_{22}^{T} & 0 & C_{22}^{T}\end{array}\right]=N$,

where $\forall s \in \mathrm{C}$ with $\operatorname{Re}[s] \geq 0$.

If the assumption 1 holds, there exist the matrices $\tilde{P}_{j j}$ such that the matrices $A_{j j}-S_{j j} \tilde{P}_{j j}$ are nonsingular, where $A_{j j}^{T} \tilde{P}_{j j}+\tilde{P}_{j j} A_{j j}-\tilde{P}_{j j} S_{j j} \tilde{P}_{j j}+Q_{j j}=0, j=1,2$. If we chose $\bar{P}_{j j}$ as $\tilde{P}_{j j}$, the unique solution of (9e) is given by $\bar{P}_{21}=0$ because the matrices $A_{j j}-S_{j j} \bar{P}_{j j}$ are nonsingular. Thus the parameter $\bar{\alpha}$ does not appear in (9). Therefore, we obtain the following 0 -order equations

$$
\begin{aligned}
& A_{s}^{T} \bar{P}_{00}+\bar{P}_{00} A_{s}-\bar{P}_{00} S_{s} \bar{P}_{00}+Q_{s}=0, \\
& \bar{P}_{j 0}^{T}=\bar{P}_{00} N_{0 j}-M_{0 j}, \\
& A_{j j}^{T} \bar{P}_{j j}+\bar{P}_{j j} A_{j j}-\bar{P}_{j j} S_{j j} \bar{P}_{j j}+Q_{j j}=0,
\end{aligned}
$$

where

$$
\begin{aligned}
A_{s}= & A_{00}+N_{01} A_{10}+N_{02} A_{20}+S_{01} M_{01}^{T}+S_{02} M_{02}^{T} \\
& +N_{01} S_{11} M_{01}^{T}+N_{02} S_{22} M_{02}^{T}, \\
S_{s}= & S_{00}+N_{01} S_{01}^{T}+S_{01} N_{01}^{T}+N_{02} S_{02}^{T}+S_{02} N_{02}^{T} \\
& +N_{01} S_{11} N_{01}^{T}+N_{02} S_{22} N_{02}^{T}, \\
Q_{s}= & Q_{00}-M_{01} A_{10}-A_{10}^{T} M_{01}^{T}-M_{02} A_{20}-A_{20}^{T} M_{02}^{T} \\
& -M_{01} S_{11} M_{01}^{T}-M_{02} S_{22} M_{02}^{T}, \\
N_{0 j}= & -D_{0 j} D_{j j}^{-1}, M_{0 j}=\bar{Q}_{0 j} D_{j j}^{-1}, \bar{Q}_{0 j}=A_{j 0}^{T} \bar{P}_{j j}+Q_{0 j}, \\
D_{00}= & A_{00}-S_{00} \bar{P}_{00}-S_{01} \bar{P}_{10}-S_{02} \bar{P}_{20}, \\
D_{0 j}= & A_{0 j}-S_{0 j} \bar{P}_{j j}, D_{j 0}=A_{j 0}-S_{0 j}^{T} \bar{P}_{00}-S_{j j} \bar{P}_{j 0}, \\
D_{j j}= & A_{j j}-S_{j j} \bar{P}_{j j}, j=1,2 .
\end{aligned}
$$

The matrices $A_{s}, S_{s}$ and $Q_{s}$ do not depend on $\bar{P}_{11}$ and $\bar{P}_{22}$ because their matrices can be computed by using $T_{p q}, p, q=0,1,2$ which is independent of $\bar{P}_{11}$ and $\bar{P}_{22}$ [5], that is,

$$
\begin{aligned}
& T_{s}=T_{00}-T_{01} T_{11}^{-1} T_{10}-T_{02} T_{22}^{-1} T_{20}=\left[\begin{array}{cc}
A_{s} & -S_{s} \\
-Q_{s} & -A_{s}^{T}
\end{array}\right], \\
& T_{00}=\left[\begin{array}{cc}
A_{00} & -S_{00} \\
-Q_{00} & -A_{00}^{T}
\end{array}\right], T_{0 j}=\left[\begin{array}{cc}
A_{0 j} & -S_{0 j} \\
-Q_{0 j} & -A_{j 0}^{T}
\end{array}\right], \\
& T_{j 0}=\left[\begin{array}{cc}
A_{j 0} & -S_{0 j}^{T} \\
-Q_{0 j}^{T} & -A_{0 j}^{T}
\end{array}\right], T_{j j}=\left[\begin{array}{cc}
A_{j j} & -S_{j j} \\
-Q_{j j} & -A_{j j}^{T}
\end{array}\right], \\
& j=1,2 .
\end{aligned}
$$

Lemma 1: Under the assumptions 1 and 2, there exist a matrix $B_{s} \in \mathbf{R}^{N \times M}, M=m_{1}+m_{2}$ and a matrix $C_{s}$ with the same dimension as $\left[\begin{array}{cc}C_{10}^{T} & C_{20}^{T}\end{array}\right]^{T}$ such that $S_{s}=$ $B_{s} R^{-1} B_{s}^{T}, Q_{s}=C_{s}^{T} C_{s}$. Moreover, the triple $\left(A_{s}, B_{s}, C_{s}\right)$ is stabilizable and detectable.

Proof: From (10a), it is easy to verify that

$$
\begin{aligned}
S_{s}= & {\left[\begin{array}{cc}
\bar{B}_{01}+N_{01} \bar{B}_{11} & \bar{B}_{02}+N_{02} \bar{B}_{22}
\end{array}\right] } \\
& \cdot\left[\begin{array}{cc}
R_{1}^{-1} & 0 \\
0 & R_{2}^{-1}
\end{array}\right]\left[\begin{array}{c}
\bar{B}_{01}^{T}+\bar{B}_{11}^{T} N_{01}^{T} \\
\bar{B}_{02}^{T}+\bar{B}_{22}^{T} N_{02}^{T}
\end{array}\right]
\end{aligned}
$$

where $\bar{B}_{0 j}=\frac{1}{\sqrt{\gamma_{j}}} B_{0 j}, \bar{B}_{j j}=\frac{1}{\sqrt{\gamma_{j}}} B_{j j}, j=1,2$.

Thus, we have $B_{s}=\left[\begin{array}{ll}\bar{B}_{01}+N_{01} \bar{B}_{11} & \bar{B}_{02}+N_{02} \bar{B}_{22}\end{array}\right]$. However, it seems difficult to find $C_{s}$ from (10a). In order to do that, we introduce a dual ARE

$$
\bar{W}_{j j} A_{j j}^{T}+A_{j j} \bar{W}_{j j}-\bar{W}_{j j} Q_{j j} \bar{W}_{j j}+S_{j j}=0, j=1,2,
$$

which admits at least a symmetric positive semidefinite solution $\tilde{W}_{j j}$ under the assumption 1 . Using (12), we find that

$$
T_{j j}=\left[\begin{array}{cc}
I_{n_{j}} & -\bar{W}_{j j} \\
0 & I_{n_{j}}
\end{array}\right]\left[\begin{array}{cc}
E_{j j} & 0 \\
-Q_{j j} & -E_{j j}^{T}
\end{array}\right]\left[\begin{array}{cc}
I_{n_{j}} & \bar{W}_{j j} \\
0 & I_{n_{j}}
\end{array}\right],
$$

where $E_{j j}=A_{j j}-Q_{j j} \tilde{W}_{j j}, j=1,2$ is nonsingular under the assumption 1. After the calculation of $T_{s}$, we arrive at another expression for $Q_{s}$, that is,

$$
\begin{aligned}
Q_{s}= & Q_{00}+L_{10}^{T} Q_{01}^{T}+Q_{01} L_{10}+L_{20}^{T} Q_{02}^{T}+Q_{02} L_{20} \\
& +L_{10}^{T} Q_{11} L_{10}+L_{20}^{T} Q_{22} L_{20}
\end{aligned}
$$

where $L_{j 0}=-E_{j j}^{-1} E_{j 0}, E_{j 0}=A_{j 0}-\tilde{W}_{j j} Q_{0 j}^{T}, j=1,2$. Hence, it is easy to find that

$$
\begin{aligned}
Q_{s} & =\left[\begin{array}{ll}
\bar{C}_{10}^{T}+L_{10}^{T} \bar{C}_{11}^{T} & \bar{C}_{20}^{T}+L_{20}^{T} \bar{C}_{22}^{T}
\end{array}\right]\left[\begin{array}{c}
\bar{C}_{10}+\bar{C}_{11} L_{10} \\
\bar{C}_{20}+\bar{C}_{22} L_{20}
\end{array}\right] \\
& =C_{s}^{T} C_{s},
\end{aligned}
$$

where $\bar{C}_{j 0}=\sqrt{\gamma_{j}} C_{j 0}, \bar{C}_{j j}=\sqrt{\gamma_{j}} C_{j j}, j=1,2$.

Let us now prove the second part of stabilizability and detectability. Note the relation

$$
\begin{aligned}
& {\left[\begin{array}{ccc}
I_{n_{0}} & -D_{01} D_{11}^{-1} & -D_{02} D_{22}^{-1} \\
0 & D_{11}^{-1} & 0 \\
0 & 0 & D_{22}^{-1}
\end{array}\right]} \\
& {\left[\begin{array}{ccccc}
s I_{n_{0}}-A_{00} & -A_{01} & -A_{02} & \bar{B}_{01} & \bar{B}_{02} \\
-A_{10} & -A_{11} & 0 & \bar{B}_{11} & 0 \\
-A_{20} & 0 & -A_{22} & 0 & \bar{B}_{22}
\end{array}\right]} \\
& {\left[\begin{array}{ccccc}
I_{n_{0}} & 0 & 0 & 0 & 0 \\
-D_{11}^{-1} A_{10} & I_{n_{1}} & 0 & \pi_{21} & 0 \\
-D_{22}^{-1} A_{20} & 0 & I_{n_{2}} & 0 & \pi_{22} \\
\pi_{11} & \pi_{31} & 0 & \pi_{41} & 0 \\
\pi_{12} & 0 & \pi_{32} & 0 & \pi_{42}
\end{array}\right]} \\
& =\left[\begin{array}{ccc}
s I_{n_{0}}-A_{00}-N_{01} A_{10}-N_{02} A_{20} & 0 & 0 \\
0 & I_{n_{1}} & 0 \\
0 & 0 & I_{n_{2}}
\end{array}\right. \\
& \left.\begin{array}{cc}
\bar{B}_{01}+N_{01} \bar{B}_{11} & \bar{B}_{02}+N_{02} \bar{B}_{22} \\
0 & 0 \\
0 & 0
\end{array}\right],
\end{aligned}
$$

where $\pi_{1 j}=-R_{j}^{-1} \bar{B}_{j j}^{T} \bar{P}_{j j} D_{11}^{-1} A_{j 0}, \pi_{2 j}=D_{j j}^{-1} \bar{B}_{j j}, \pi_{3 j}=$ $R_{j}^{-1} \bar{B}_{j j}^{T} \bar{P}_{j j}, \pi_{4 j}=I_{n_{j}}+R_{j}^{-1} \bar{B}_{j j}^{T} \bar{P}_{j j} D_{j j}^{-1} \bar{B}_{j j}, \quad j=1,2$. Hence, the couple $\left(A_{s}, B_{s}\right)$ is stabilizable if and only if $\operatorname{rank}\left[s I_{n_{0}}-A_{00}-N_{01} A_{10}-N_{02} A_{20} B_{s}\right]=n_{0}, \forall s \in \mathrm{C}$. In other words, the matrix pair $\left(A_{00}+N_{01} A_{10}+N_{02} A_{20}, B_{s}\right)$ is stabilizable. Since $A_{s}=A_{00}+N_{01} A_{10}+N_{02} A_{20}+B_{s} \mathcal{K}$ and the feedback $\mathcal{K}$ does not change the stabilizable property of 


\section{IECON'01: The 27th Annual Conference of the IEEE Industrial Electronics Society}

$\left(A_{00}+N_{01} A_{10}+N_{02} A_{20}, B_{s}\right)$, we arrive at the conclusion that the matrix pair $\left(A_{s}, B_{s}\right)$ is also stabilizable. Similarly, we can prove that $\left(A_{s}^{T}, C_{s}^{T}\right)$ is detectable if and only if the assumption 2 is satisfied. The detail is omitted for brevity. Thereby, we have finished the proof of Lemma 1.

The following theorem will establish the relation between $P_{\mathcal{E}}$ and reduced-order solutions (10).

Theorem 1: Under the assumptions 1 and 2, there exist small $\varepsilon_{j}^{*}, j=1,2$ such that for all $\varepsilon_{j} \in\left(0, \varepsilon_{j}^{*}\right)$, the MARE (6) admits a symmetric positive semidefinite stabilizing solution $P_{\mathcal{E}}$ which can be written as

$$
\begin{gathered}
P_{\mathcal{E}}= \\
{\left[\begin{array}{ccc}
\bar{P}_{00}+\mathcal{F}_{00} & \varepsilon_{1}\left(\bar{P}_{10}+\mathcal{F}_{10}\right)^{T} & \varepsilon_{2}\left(\bar{P}_{20}+\mathcal{F}_{20}\right)^{T} \\
\varepsilon_{1}\left(\bar{P}_{10}+\mathcal{F}_{10}\right) & \varepsilon_{1}\left(\bar{P}_{11}+\mathcal{F}_{11}\right) & \sqrt{\varepsilon_{1} \varepsilon_{2}} \mathcal{F}_{21}^{T} \\
\varepsilon_{2}\left(\bar{P}_{20}+\mathcal{F}_{20}\right) & \sqrt{\varepsilon_{1} \varepsilon_{2}} \mathcal{F}_{21} & \varepsilon_{2}\left(\bar{P}_{22}+\mathcal{F}_{22}\right)
\end{array}\right],}
\end{gathered}
$$

where $\mathcal{F}_{p q}(\|\mu\|)=O(\|\mu\|)$ with

$$
p q=00,10,20,11,21,22, \mu=\left[\varepsilon_{1} \varepsilon_{2}\right] .
$$

Proof: We apply the implicit function theorem [4] to (7). To do so, it is enough to show that the corresponding Jacobian is nonsingular at $\varepsilon_{j}=0, j=1,2$. It can be shown, after some algebra, that the Jacobian of (7) in the limit is given by

$$
\begin{aligned}
J & =\nabla \mathbf{F} \\
& =\left.\frac{\partial \operatorname{vec}\left(f_{1}, f_{2}, f_{3}, f_{4}, f_{5}, f_{6}\right)}{\partial \operatorname{vec}\left(P_{00}, P_{10}, P_{20}, P_{11}, P_{21}, P_{22}\right)^{T}}\right|_{\mu=\mu_{0}, \mathcal{P}=\mathcal{P}_{0}} \\
& =\left[\begin{array}{cccccc}
J_{00} & J_{01} & J_{02} & 0 & 0 & 0 \\
J_{10} & J_{11} & 0 & J_{13} & J_{14} & 0 \\
J_{20} & 0 & J_{22} & 0 & J_{24} & J_{25} \\
0 & 0 & 0 & J_{33} & 0 & 0 \\
0 & 0 & 0 & 0 & J_{44} & 0 \\
0 & 0 & 0 & 0 & 0 & J_{55}
\end{array}\right]
\end{aligned}
$$

where vec denotes an ordered stack of the columns of its matrix [7] and

$$
\begin{aligned}
& \mu=\left[\begin{array}{ll}
\varepsilon_{1} & \varepsilon_{2}
\end{array}\right], \mu_{0}=\left[\begin{array}{ll}
0 & 0
\end{array}\right], \\
& \mathcal{P}=\left(P_{00}, P_{10}, P_{20}, P_{11}, P_{21}, P_{22}\right), \\
& \mathcal{P}_{0}=\left(\bar{P}_{00}, \bar{P}_{10}, \bar{P}_{20} \bar{P}_{11}, 0, \bar{P}_{22}\right), \\
& J_{00}=\left(I_{n_{0}} \otimes D_{00}^{T}\right) U_{n_{0} n_{0}}+D_{00}^{T} \otimes I_{n_{0}}, \\
& J_{0 j}=\left(I_{n_{0}} \otimes D_{j 0}^{T}\right) U_{n_{0} n_{1}}+D_{j 0}^{T} \otimes I_{n_{0}}, \\
& J_{j 0}=D_{0 j}^{T} \otimes I_{n_{0}}, J_{j j}=D_{j j}^{T} \otimes I_{n_{0}}, \\
& J_{13}=D_{10}^{T} \otimes I_{n_{1}}, J_{14}=\sqrt{\bar{\alpha}}\left(D_{20}^{T} \otimes I_{n_{1}}\right) U_{n_{1} n_{2}}, \\
& J_{24}=\frac{1}{\sqrt{\bar{\alpha}}} D_{10}^{T} \otimes I_{n_{2}}, J_{25}=D_{20}^{T} \otimes I_{n_{2}}, \\
& J_{33}=\left(I_{n_{1}} \otimes D_{11}^{T}\right) U_{n_{1} n_{1}}+D_{11}^{T} \otimes I_{n_{1}}, \\
& J_{44}=\sqrt{\bar{\alpha}} D_{22}^{T} \otimes I_{n_{1}}+\frac{1}{\sqrt{\bar{\alpha}}} I_{n_{2}} \otimes D_{11}^{T}, \\
& J_{55}=\left(I_{n_{2}} \otimes D_{22}^{T}\right) U_{n_{2} n_{2}}+D_{22}^{T} \otimes I_{n_{2}}, j=1,2
\end{aligned}
$$

where $\otimes$ denotes Kronecker products and $U_{n_{j} n_{j}}, j=$ $0,1,2$ is the permutation matrix in Kronecker matrix sense $[7]$.
The Jacobian (15) can be expressed as

$$
\begin{aligned}
\operatorname{det} J= & \operatorname{det} J_{11} \cdot \operatorname{det} J_{22} \cdot \operatorname{det} J_{33} \cdot \operatorname{det} J_{44} \cdot \operatorname{det} J_{55} \\
& \cdot \operatorname{det}\left[I_{n_{0}} \otimes D_{0}^{T} U_{n_{0} n_{0}}+D_{0}^{T} \otimes I_{n_{0}}\right]
\end{aligned}
$$

where $D_{0} \equiv D_{00}-D_{01} D_{11}^{-1} D_{10}-D_{02} D_{22}^{-1} D_{20}$. Obviously, $J_{j j}, j=1, \cdots, 5$ are nonsingular because the matrices $D_{j j}=A_{j j}-S_{j j} \bar{P}_{j j}, j=1,2$ are nonsingular under the assumption 1. After some straightforward algebra but tedious, we see that $A_{s}-S_{s} \bar{P}_{00}=D_{00}-D_{01} D_{11}^{-1} D_{10}-$ $D_{02} D_{22}^{-1} D_{20}=D_{0}$. Therefore, the matrix $D_{0}$ is nonsingular if the assumption 2 holds. Thus, $\operatorname{det} J \neq 0$, i.e., $J$ is nonsingular at $(\mu, \mathcal{P})=\left(\mu_{0}, \mathcal{P}_{0}\right)$. The conclusion of Theorem 1 is obtained directly by using the implicit function theorem.

With respect to the results of [4], we do not require singularity of $A_{j j}, j=1,2$. Moreover, we believe that our proof is very directly. Thus, our new results are applicable to more realistic MSPS.

\section{Pareto near-Optimal strategy}

Our attention is focused on the near-optimal strategy design. Such a strategy is obtained by using the reducedorder solutions (10). Hence, we can get the Pareto nearoptimal strategy

$$
u_{\mathrm{app} j}(t)=-\frac{1}{\gamma_{j}} R_{j}^{-1} B_{j}^{T} \bar{P} x(t), \quad j=1,2,
$$

where

$$
\begin{aligned}
& \bar{P}=\left[\begin{array}{ccc}
\bar{P}_{00} & 0 & 0 \\
\bar{P}_{10} & \bar{P}_{11} & 0 \\
\bar{P}_{20} & 0 & \bar{P}_{22}
\end{array}\right], \Phi_{\mathcal{E}}=\left[\begin{array}{ccc}
I_{n_{0}} & 0 & 0 \\
0 & \varepsilon_{1} I_{n_{1}} & 0 \\
0 & 0 & \varepsilon_{2} I_{n_{2}}
\end{array}\right], \\
& \bar{P}_{\mathcal{E}}=\Phi_{\mathcal{E}} \bar{P}, B_{j}=\Phi_{\mathcal{E}} B_{j \mathcal{E}}, j=1,2 .
\end{aligned}
$$

Theorem 2: Under the assumptions 1 and 2, the use of the near-optimal strategy (17) results in $\bar{J}_{j}$ satisfying

$$
\bar{J}_{j}=J_{j}^{*}+O(\|\mu\|), \quad j=1,2,
$$

where the value of the actual cost is

$$
\bar{J}_{j}=\frac{1}{2} x(0)^{T} Y_{j \mathcal{E}} x(0), j=1,2,
$$

and $Y_{j \mathcal{E}}$ is a positive semidefinite solution of the multiparameter algebraic Lyapunov equation (MALE)

$$
\begin{aligned}
& Y_{j \mathcal{E}}\left(A_{\mathcal{E}}-S_{\mathcal{E}} \bar{P}_{\mathcal{E}}\right)+\left(A_{\mathcal{E}}-S_{\mathcal{E}} \bar{P}_{\mathcal{E}}\right)^{T} Y_{j \mathcal{E}} \\
& \quad+Q_{j}+\frac{1}{\gamma_{j}^{2}} \bar{P}_{\mathcal{E}} S_{j \mathcal{E}} \bar{P}_{\mathcal{E}}=0, j=1,2 .
\end{aligned}
$$

Proof: When the Pareto optimal strategy (5) is given, they result in

$$
J_{j}^{*}=\frac{1}{2} x(0)^{T} X_{j \mathcal{E}} x(0), j=1,2,
$$

where $X_{j \mathcal{E}}$ is a positive semidefinite solution of the MALE

$$
\begin{array}{r}
X_{j \mathcal{E}}\left(A_{\mathcal{E}}-S_{\mathcal{E}} P_{\mathcal{E}}\right)+\left(A_{\mathcal{E}}-S_{\mathcal{E}} P_{\mathcal{E}}\right)^{T} X_{j \mathcal{E}} \\
+Q_{j}+\frac{1}{\gamma_{j}^{2}} P_{\mathcal{E}} S_{j \mathcal{E}} P_{\mathcal{E}}=0, j=1,2 .
\end{array}
$$




\section{IECON'01: The 27th Annual Conference of the IEEE Industrial Electronics Society}

In order to calculate the loss of performance $\left(\bar{J}_{j}-J_{j}^{*}\right)$, we subtract (22) from (20) and obtain the MALE for $Z_{j \mathcal{E}}=$ $Y_{j \mathcal{E}}-X_{j \mathcal{E}}$

$$
\begin{aligned}
Z_{j \mathcal{E}}( & \left.A_{\mathcal{E}}-S_{\mathcal{E}} \bar{P}_{\mathcal{E}}\right)+\left(A_{\mathcal{E}}-S_{\mathcal{E}} \bar{P}_{\mathcal{E}}\right)^{T} Z_{j \mathcal{E}}+\frac{1}{\gamma_{j}^{2}} \bar{P}_{\mathcal{E}} S_{j \mathcal{E}} \bar{P}_{\mathcal{E}} \\
& -\frac{1}{\gamma_{j}^{2}} P_{\mathcal{E}} S_{j \mathcal{E}} P_{\mathcal{E}}+X_{j \mathcal{E}} S_{j \mathcal{E}}\left(P_{\mathcal{E}}-\bar{P}_{\mathcal{E}}\right) \\
& +\left(P_{\mathcal{E}}-\bar{P}_{\mathcal{E}}\right)^{T} S_{j \mathcal{E}} X_{j \mathcal{E}}=0, j=1,2 .
\end{aligned}
$$

Using the result established in (14), it is easy to verify that

$$
\left\|\bar{P}_{\mathcal{E}}-P_{\mathcal{E}}\right\|=O(\|\mu\|) .
$$

It follows from (24) that

$$
\begin{aligned}
& Z_{j \mathcal{E}}\left(A_{\mathcal{E}}-S_{\mathcal{E}} \bar{P}_{\mathcal{E}}\right)+\left(A_{\mathcal{E}}-S_{\mathcal{E}} \bar{P}_{\mathcal{E}}\right)^{T} Z_{j \mathcal{E}}+O(\|\mu\|)=0, \\
& j=1,2 .
\end{aligned}
$$

Since $D_{11}, D_{22}$ and $D_{0}$ are stable, $A_{\mathcal{E}}-S_{\mathcal{E}} \bar{P}_{\mathcal{E}}$ is stable from Theorem 1 [1]. Hence

$$
Z_{j \mathcal{E}}=O(\|\mu\|), j=1,2
$$

implying (18).

In the rest of this section, we will show that the Pareto near-optimal strategy (17) is equivalent to the existing composite strategy [1] as the special case. Let $A_{j j}, j=1,2$ of (1) be nonsingular. In this case, the composite strategy is

$$
\begin{gathered}
u_{\mathrm{com} j}(t)=-\frac{1}{\gamma_{j}} R_{j}^{-1} B_{j}^{T} X x(t) \\
=-\frac{1}{\gamma_{j}} R_{j}^{-1} B_{j}^{T}\left[\begin{array}{ccc}
X_{00} & 0 & 0 \\
X_{10} & X_{11} & 0 \\
X_{20} & 0 & X_{22}
\end{array}\right] x(t), j=1,2 .
\end{gathered}
$$

In the above, $X_{00}$ is the unique stabilizing positive semidefinite symmetric solution of the following ARE

$$
\begin{gathered}
\left(A_{r}-B_{r} R_{r}^{-1} E_{r}^{T} C_{r}\right)^{T} X_{00}+X_{00}\left(A_{r}-B_{r} R_{r}^{-1} E_{r}^{T} C_{r}\right) \\
-X_{00} B_{r} R_{r}^{-1} B_{r}^{T} X_{00}+C_{r}^{T}\left(I_{n_{0}}-E_{r} R_{r}^{-1} E_{r}^{T}\right) C_{r}=0
\end{gathered}
$$

where

$$
\begin{aligned}
& R_{r}=R+E_{r}^{T} E_{r}, A_{r}=A_{00}-A_{01} A_{11}^{-1} A_{10}-A_{02} A_{22}^{-1} A_{20}, \\
& B_{r}=\left[\begin{array}{cc}
\bar{B}_{01}-A_{01} A_{11}^{-1} \bar{B}_{11} & \bar{B}_{02}-A_{02} A_{22}^{-1} \bar{B}_{22}
\end{array}\right] \text {, } \\
& C_{r}=\left[\begin{array}{c}
\bar{C}_{10}-\bar{C}_{11} A_{11}^{-1} A_{10} \\
\bar{C}_{20}-\bar{C}_{22} A_{22}^{-1} A_{20}
\end{array}\right], \\
& E_{r}=-\left[\begin{array}{cc}
\bar{C}_{11} A_{11}^{-1} \bar{B}_{11} & 0 \\
0 & \bar{C}_{22} A_{22}^{-1} \bar{B}_{22}
\end{array}\right] \text {. }
\end{aligned}
$$

$X_{j j}, j=1,2$ are the unique stabilizing positive semidefinite solution of the following AREs

$$
A_{j j}^{T} X_{j j}+X_{j j} A_{j j}-X_{j j} S_{j j} X_{j j}+Q_{j j}=0, j=1,2
$$

and $X_{j 0}$ are

$$
\begin{aligned}
X_{j 0}^{T}= & {\left[X_{00}\left(S_{0 j} X_{j j}-A_{0 j}\right)-\left(A_{j 0}^{T} X_{j j}\right.\right.} \\
& \left.\left.+Q_{0 j}\right)\right]\left(A_{j j}-S_{j j} X_{j j}\right)^{-1}, j=1,2 .
\end{aligned}
$$

Theorem 3: Under the assumptions 1 and 2, the following identities hold.

$$
X_{j j}=\bar{P}_{j j}, X_{j 0}=\bar{P}_{j 0}, X_{00}=\bar{P}_{00}, j=1,2,
$$

and hence the resulting near-optimal controller (17) is the same as the composite optimal controller (27).

Proof: It can be carried out via a similar technique used in [8]. Firstly, comparing (29) with (10c) yields $X_{j j}=$ $\bar{P}_{j j}, j=1,2$ directly. Secondly, comparing (30) with (10b) and noting that $X_{j j}=\bar{P}_{j j}$, we have the conclusion that $X_{j 0}=\bar{P}_{j 0}, j=1,2$ if $X_{00}=\bar{P}_{00}$. Therefore, the remainder of the proof is to show that $X_{00}=\bar{P}_{00}$. In order to do that, we only need to show that the ARE (28) and (10a) are the same equations, that is,

$$
\begin{aligned}
& A_{r}-B_{r} R_{r}^{-1} E_{r}^{T} C_{r}=A_{s}, \\
& B_{r} R_{r}^{-1} B_{r}^{T}=S_{s}, \\
& C_{r}^{T}\left(I_{n_{0}}-E_{r} R_{r}^{-1} E_{r}^{T}\right) C_{r}=Q_{s} .
\end{aligned}
$$

Before showing these relations, let us define (pp.115, [6])

$$
\begin{aligned}
H & =I_{n_{1}+n_{2}} \\
& +\left[\begin{array}{cc}
R_{1}^{-1} \bar{B}_{11}^{T} \bar{P}_{11} D_{11}^{-1} \bar{B}_{11} & 0 \\
0 & R_{2}^{-1} \bar{B}_{22}^{T} \bar{P}_{22} D_{22}^{-1} \bar{B}_{22}
\end{array}\right]
\end{aligned}
$$

Then,

$$
\begin{aligned}
H^{-1} & =I_{n_{1}+n_{2}} \\
& -\left[\begin{array}{cc}
R_{1}^{-1} \bar{B}_{11}^{T} \bar{P}_{11} A_{11}^{-1} \bar{B}_{11} & 0 \\
0 & R_{2}^{-1} \bar{B}_{22}^{T} \bar{P}_{22} A_{22}^{-1} \bar{B}_{22}
\end{array}\right] .
\end{aligned}
$$

Thus, using (34) and the ARE (10c) we have

$$
H^{-T} R H^{-1}=R+E_{r}^{T} E_{r}=R_{r} \Leftrightarrow H R^{-1} H^{T}=R_{r}^{-1} .
$$

Let us further introduce six useful identities.

$$
\begin{aligned}
& A_{j j}^{-1}+A_{j j}^{-1} S_{j j} \bar{P}_{j j} D_{j j}^{-1}=D_{j j}^{-1}, \\
& A_{j j}^{-1}+D_{j j}^{-1} S_{j j} \bar{P}_{j j} A_{j j}^{-1}=D_{j j}^{-1}, \\
& I_{n_{j}}+S_{j j} \bar{P}_{j j} D_{j j}^{-1}=A_{j j} D_{j j}^{-1}, \\
& I_{n_{j}}+\bar{P}_{j j} S_{j j} D_{j j}^{-T}=A_{j j}^{T} D_{j j}^{-T}, \\
& Q_{0 j}^{T}-Q_{j j} A_{j j}^{-1} A_{j 0}=\bar{Q}_{0 j}^{T}+D_{j j}^{T} \bar{P}_{j j} A_{j j}^{-1} A_{j 0}, \\
& -D_{0 j}+N_{0 j} S_{j j} \bar{P}_{j j}=N_{0 j} A_{j j}, j=1,2 .
\end{aligned}
$$

Then, we get

$$
\begin{aligned}
& -B_{r} R_{r}^{-1} E_{r} \\
= & {\left[\begin{array}{cc}
S_{01}-A_{01} A_{11}^{-1} S_{11} & S_{02}-A_{02} A_{22}^{-1} S_{22}
\end{array}\right] } \\
& \cdot\left[\begin{array}{c}
D_{11}^{-T} \bar{C}_{11}^{T}+\bar{P}_{11} D_{11}^{-1} S_{11} D_{11}^{-T} \bar{C}_{11}^{T} \\
0 \\
0 \\
\\
\quad D_{22}^{-T} \bar{C}_{22}^{T}+\bar{P}_{22} D_{22}^{-1} S_{22} D_{22}^{-T} \bar{C}_{22}^{T}
\end{array}\right] \cdot
\end{aligned}
$$

Hence,

$$
A_{r}-B_{r} R_{r}^{-1} E_{r}^{T} C_{r}
$$


IECON'01: The 27th Annual Conference of the IEEE Industrial Electronics Society

$$
\begin{aligned}
& =A_{00}-A_{01} A_{11}^{-1} A_{10}-A_{02} A_{22}^{-1} A_{20} \\
& +\left[\begin{array}{ll}
S_{01}-A_{01} A_{11}^{-1} S_{11} & S_{02}-A_{02} A_{22}^{-1} S_{22}
\end{array}\right] \\
& \cdot\left[\begin{array}{c}
\left(I_{n_{1}}+\bar{P}_{11} D_{11}^{-1} S_{11}\right) D_{11}^{-T}\left(Q_{01}^{T}-Q_{11} A_{11}^{-1} A_{10}\right) \\
\left(I_{n_{2}}+\bar{P}_{22} D_{22}^{-1} S_{22}\right) D_{22}^{-T}\left(Q_{02}^{T}-Q_{22} A_{22}^{-1} A_{20}\right)
\end{array}\right] \\
& =A_{00}-A_{01} A_{11}^{-1} A_{10}-A_{02} A_{22}^{-1} A_{20} \\
& +\left(S_{01}+N_{01} S_{11}\right) D_{11}^{-T}\left(\bar{Q}_{01}^{T}+D_{11}^{T} \bar{P}_{11} A_{11}^{-1} A_{10}\right) \\
& +\left(S_{02}+N_{02} S_{22}\right) D_{22}^{-T}\left(\bar{Q}_{02}^{T}+D_{22}^{T} \bar{P}_{22} A_{22}^{-1} A_{20}\right) \\
& =A_{00}+N_{01} S_{11} M_{01}^{T}+N_{02} S_{22} M_{02}^{T} \\
& \text { - } D_{01} A_{11}^{-1} A_{10}-D_{02} A_{22}^{-1} A_{20} \\
& +N_{01} S_{11} \bar{P}_{11} A_{11}^{-1} A_{10}+N_{02} S_{22} \bar{P}_{22} A_{22}^{-1} A_{20} \\
& +S_{01} M_{01}^{T}+S_{02} M_{02}^{T} \text {, }
\end{aligned}
$$

where the identities (36) have also been used to simplify the expressions. After expanding $A_{s}$ of (10a), we arrive at the conclusion that $A_{s}$ is the same as (38), which proves (32a). Now, considering (32b), we have

$$
\begin{aligned}
& B_{r} H \\
= & {\left[\begin{array}{ll}
\bar{B}_{01} & \bar{B}_{02}
\end{array}\right]-\left[\begin{array}{ll}
D_{01} D_{11}^{-1} \bar{B}_{11} & D_{02} D_{22}^{-1} \bar{B}_{22}
\end{array}\right] } \\
& +\left[\begin{array}{ll}
A_{01} D_{11}^{-1} \bar{B}_{11} & A_{02} D_{22}^{-1} \bar{B}_{22}
\end{array}\right] \\
& -\left[\begin{array}{cc}
A_{01} A_{11}^{-1} \bar{B}_{11} R_{1}^{-1} \bar{B}_{11}^{T} \bar{P}_{11} D_{11}^{-1} \bar{B}_{11} \\
& A_{02} A_{22}^{-1} \bar{B}_{22} R_{2}^{-1} \bar{B}_{22}^{T} \bar{P}_{22} D_{22}^{-1} \bar{B}_{22}
\end{array}\right] \\
& -\left[\begin{array}{ll}
A_{01} A_{11}^{-1} \bar{B}_{11} & A_{02} A_{22}^{-1} \bar{B}_{22}
\end{array}\right] \\
= & {\left[\begin{array}{lll}
\bar{B}_{01} & \bar{B}_{02}
\end{array}\right]+\left[\begin{array}{ll}
N_{01} \bar{B}_{11} & N_{02} \bar{B}_{22}
\end{array}\right] } \\
= & {\left[\begin{array}{ll}
\bar{B}_{01}+N_{01} \bar{B}_{11} & \bar{B}_{02}+N_{02} \bar{B}_{22}
\end{array}\right]=B_{s} . }
\end{aligned}
$$

Hence, using (39), we have

$$
B_{r} R_{0}^{-1} B_{r}^{T}=B_{r} H R^{-1} H^{T} B_{r}^{T}=B_{s} R^{-1} B_{s}^{T}=S_{s},
$$

which proves $(32 \mathrm{~b})$.

Finally, using the identities of (36), it is straightforward but tedious to verify that

$$
\begin{aligned}
& E_{r} R_{r}^{-1} E_{r}^{T} \\
& =\left[\begin{array}{cc}
\bar{C}_{11} D_{11}^{-1} S_{11} D_{11}^{-T} \bar{C}_{11}^{T} & 0 \\
0 & \bar{C}_{22} D_{22}^{-1} S_{22} D_{22}^{-T} \bar{C}_{22}^{T}
\end{array}\right]
\end{aligned}
$$

Moreover, we get

$$
\begin{aligned}
C_{r}^{T} C_{r}= & Q_{00}-\bar{Q}_{01} A_{11}^{-1} A_{10}-A_{10}^{T} A_{11}^{-T} \bar{Q}_{01}^{T} \\
& -\bar{Q}_{02} A_{22}^{-1} A_{20}-A_{20}^{T} A_{22}^{-T} \bar{Q}_{02}^{T} \\
& +A_{10}^{T} A_{11}^{-T} \bar{P}_{11} S_{11} \bar{P}_{11} A_{11}^{-1} A_{10} \\
& +A_{20}^{T} A_{22}^{-T} \bar{P}_{22} S_{22} \bar{P}_{22} A_{22}^{-1} A_{20} .
\end{aligned}
$$

Since $-Q_{j j}=A_{j j}^{T} \bar{P}_{j j}+\bar{P}_{j j} A_{j j}-\bar{P}_{j j} S_{j j} \bar{P}_{j j}, j=1,2$, it follows that

$$
\begin{aligned}
& C_{r}^{T} C_{r}-C_{r}^{T} E_{r} R_{r}^{-1} E_{r}^{T} C_{r} \\
= & C_{r}^{T} C_{r}-\bar{Q}_{01} D_{11}^{-1} A_{10}-A_{10}^{T} D_{11}^{-T} \bar{Q}_{01}^{T}+\bar{Q}_{01} A_{11}^{-1} A_{10} \\
& +A_{10}^{T} A_{11}^{-T} \bar{Q}_{01}^{T}-\bar{Q}_{01} D_{11}^{-1} S_{11} D_{11}^{-T} \bar{Q}_{01}^{T} \\
& -A_{10}^{T} A_{11}^{-T} \bar{P}_{11} S_{11} \bar{P}_{11} A_{11}^{-1} A_{10}-\bar{Q}_{02} D_{22}^{-1} A_{20} \\
& -A_{20}^{T} D_{22}^{-T} \bar{Q}_{02}^{T}+\bar{Q}_{02} A_{22}^{-1} A_{20}+A_{20}^{T} A_{22}^{-T} \bar{Q}_{02}^{T}
\end{aligned}
$$

$$
\begin{aligned}
& -\bar{Q}_{02} D_{22}^{-1} S_{22} D_{22}^{-T} \bar{Q}_{02}^{T}-A_{20}^{T} A_{22}^{-T} \bar{P}_{22} S_{22} \bar{P}_{22} A_{22}^{-1} A_{20} \\
= & Q_{00}-\bar{Q}_{01} A_{11}^{-1} A_{10}-A_{10}^{T} A_{11}^{-T} \bar{Q}_{01}^{T}-\bar{Q}_{02} A_{22}^{-1} A_{20} \\
& -A_{20}^{T} A_{22}^{-T} \bar{Q}_{02}^{T}-\bar{Q}_{01} D_{11}^{-1} A_{10}-A_{10}^{T} D_{11}^{-T} \bar{Q}_{01}^{T} \\
& +\bar{Q}_{01} A_{11}^{-1} A_{10}+A_{10}^{T} A_{11}^{-T} \bar{Q}_{01}^{T}-\bar{Q}_{01} D_{11}^{-1} S_{11} D_{11}^{-T} \bar{Q}_{01}^{T} \\
& -\bar{Q}_{02} D_{22}^{-1} A_{20}-A_{20}^{T} D_{22}^{-T} \bar{Q}_{02}^{T}+\bar{Q}_{02} A_{22}^{-1} A_{20} \\
& +A_{20}^{T} A_{22}^{-T} \bar{Q}_{02}^{T}-\bar{Q}_{02} D_{22}^{-1} S_{22} D_{22}^{-T} \bar{Q}_{02}^{T} .
\end{aligned}
$$

On the other hand, expanding $Q_{s}$ of (10a) and noting $X_{j j}=\bar{P}_{j j}$ lead to the conclusion that $Q_{s}$ is the same as (43), which proves $(32 \mathrm{c})$. In consequence, we have $X_{00}=$ $\bar{P}_{00}$, hence, $X_{j 0}=\bar{P}_{j 0}, j=1,2$. The proof of Theorem 3 is completed.

\section{CONClusions}

In this paper, we have studied the Pareto near-optimal strategy associated with the MSPS. We have proposed the new Pareto near-optimal strategy. We have shown that an $O(\|\mu\|)$ accuracy strategy achieves the cost functional $J_{j}^{*}+$ $O(\|\mu\|)$. Moreover, we have also shown that the resulting strategy is equivalent to the existing one. Thus, our new results are applicable to more realistic MSPS. In addition, it is easy to apply our analysis to the optimal regulator problem for the MSPS because the solution of such problem is a special case of the Pareto optimal strategy when the decision makers agree on a choice of a weighting factors.

\section{REFERENCES}

[1] H.K.Khalil and P.V.Kokotović, "Control strategies for decision makers using different models of the same system," IEEE Trans. Automatic Control, vol.23, 1978, pp.289-298.

[2] H.K.Khalil and P.V.Kokotović, "Control of linear systems with multiparameter singular perturbations," Automatica, vol.15, 1979, pp.197-207.

[3] H.K.Khalil, "Stabilization of multiparameter singularly perturbed systems," IEEE Transactions on Automatic Control, vol.24, 1979, pp.790-791.

[4] Z.Gajic, "The existence of a unique and bounded solution of the algebraic Riccati equation of multimodel estimation and control problems," Syst. Control Lett., vol.10, 1988, pp.185-190.

[5] C.Coumarbatch and Z.Gajic, "Exact decomposition of the algebraic Riccati equation of deterministic multimodeling optimal control problems," IEEE Trans. Automatic Control, vol.45, 2000, pp.790-794.

[6] P.V.Kokotović, H.K.Khalil and J.O'Reilly, Singular perturbation methods in control: analysis and design. Academic Press, New York: 1986.

[7] J.R.Magnus and H.Neudecker, Matrix Differential Calculus with Applications in Statistics and Econometrics, John Wiley and Sons, New York: 1999.

[8] H.Xu, H.Mukaidani and K.Mizukami, "New method for composite optimal control of singularly perturbed systems," Int. J. Systems Science, vol.28, 1997, pp.161-172.

[9] H.Mukaidani, H.Xu and K.Mizukami, Recursive computation of Pareto optimal strategy for multiparameter singularly perturbed systems, Dynamics of Continuous, Discrete and Impulsive Systems, vol.8, 2001. (to appear) 